\title{
Perspective
}

PERSPECTIVE Actualité en histoire de l'art

2| 2006

La Suisse/Période moderne

\section{Les colloques de la relève suisse en histoire de l'art : une initiative du corps intermédiaire universitaire}

Julia Gelshorn

\section{(2) OpenEdition Journals}

\section{Édition électronique}

URL : http://journals.openedition.org/perspective/377

DOI : $10.4000 /$ perspective. 377

ISSN : 2269-7721

\section{Éditeur}

Institut national d'histoire de l'art

\section{Édition imprimée}

Date de publication : 30 juillet 2006

Pagination : 267-270

ISSN : $1777-7852$

\section{Référence électronique}

Julia Gelshorn, « Les colloques de la relève suisse en histoire de l'art : une initiative du corps intermédiaire universitaire », Perspective [En ligne], 2 | 2006, mis en ligne le 31 mars 2018, consulté le 01 octobre 2020. URL : http://journals.openedition.org/perspective/377 ; DOI : https://doi.org/

10.4000/perspective.377 


\title{
Les colloques de la relève suisse en histoire de l'art : une initiative du corps intermédiaire universitaire
}

\author{
Julia Gelshorn
}

1 Sous le titre La soupe et les nuages, les membres du corps intermédiaire du département d'histoire de l'art de l'Université de Lausanne ont lancé en 1997 un colloque qui ne s'adressait qu'aux jeunes chercheurs afin de leur donner l'occasion de présenter leurs projets de recherche à la communauté scientifique, de nouer des contacts et de discuter de leur situation en Suisse. Le titre La soupe et les nuages fait référence au poème en prose de Charles Baudelaire, dans lequel un artiste est brusquement arraché à sa contemplation des nuages, ces "merveilleuses constructions de l'impalpable», pour revenir à la réalité. Ce poème a servi aux organisateurs du colloque juste pour illustrer les deux pôles extrêmes qui déterminent souvent la situation des jeunes historiens de l'art en Suisse: "d'un côté les idéaux de la recherche, de l'autre les contingences quotidiennes et les difficultés toujours plus grandes à trouver le soutien nécessaire pour continuer $»^{1}$.

2 Dans les trois langues officielles de la Suisse (l'allemand, le français et l'italien), vingtquatre jeunes historiens de l'art ont présenté leurs projets. Brassant l'art occidental du Moyen Âge au $\mathrm{xx}^{\mathrm{e}}$ siècle, ils ont témoigné d'une grande diversité thématique et méthodologique. Le colloque, d'une durée de trois jours, s'est terminé par une table ronde qui était l'occasion de discuter aussi bien de la formation en histoire de l'art et des perspectives professionnelles que du potentiel et des problèmes de la discipline.

Le succès a été tel que, les années suivantes, les autres départements universitaires suisses d'histoire de l'art ont repris cette initiative en organisant des colloques annuels pour jeunes chercheurs - initiative qui se poursuit toujours. À Lausanne a succédé Berne, puis Zurich, Bâle, Fribourg, Genève et Neuchâtel, jusqu'à ce qu'en 2004-2005 Lausanne recommence ce cycle, suivi en 2005 par Berne et en 2006 par Zurich. Les colloques se limitent désormais à deux jours, financés d'un côté par l'université d'accueil et de l'autre par l'Académie suisse des sciences humaines et sociales (ASSH) et 
par l'Association suisse des historiens et Historiennes de l'Art (ASHHA) qui ont reconnu l'importance de ces rencontres².

4 Pourquoi, voudrait-on demander, le modèle de ce colloque pour jeunes chercheurs a-til eu tellement de succès? Quel avait été à l'origine le besoin urgent qui a été apparemment satisfait par cette initiative?

Dès 1997, la nécessité de créer des réseaux et de professionnaliser la discipline a fait l'objet d'une première table ronde, et cette nécessité reste toujours valable. En vue des réformes universitaires après la Déclaration de Bologne (qui met en place le système du LMD), les résolutions du premier colloque de 1997 ont même gagné en actualité3.

6 En 1997, la discussion a porté, entre autres, sur la préparation de la relève grâce à un enseignement d'histoire de l'art au lycée, le manque de compatibilité des études entre la Suisse et les autres pays d'Europe, les différences entre les systèmes romand et allemand au niveau de la thèse de doctorat et de l'habilitation, les aides financières sous forme d'allocations et de bourses et les possibilités d'un échange national en histoire de l'art. En 2004, à la fin du premier cycle des colloques pour jeunes chercheurs, la table ronde "À propos de la relève » organisée à Neuchâtel a réévalué ces intentions de façon rétrospective et prospective ${ }^{4}$. D'où il est apparu que, même sept ans plus tard, les résolutions de Lausanne n'ont rien perdu de leur actualité. Comme l'histoire de l'art est très peu enseignée au lycée et que la formation des professeurs pour l'enseignement secondaire n'a que rarement lieu à l'université, la relève en histoire de l'art fait défaut. Au plus haut niveau, ce manque se reflète dans le petit nombre de doctorants inscrits en Suisse et la faible répartition des postes universitaires germanophones attribués aux Suisses qui reste nettement en-dessous de $50 \%$. De même, explicite dans la Déclaration de Bologne, l'unification des études en licence/ master pour faciliter la mobilité des étudiants a déjà échoué au niveau national car les programmes des universités sont très différents les uns des autres. Cela est aussi valable pour le troisième cycle et la carrière de chercheur puisque le doctorat romand et l'habilitation de la Suisse allemande sont toujours incompatibles, tandis que l'Allemagne supprime l'habilitation et la France la réinstaure. Enfin, le soutien de la jeune génération suisse se limite surtout au Fonds national suisse de la recherche scientifique (SNF).

7 Ainsi, les colloques de la relève suisse ne sont-ils pas uniquement un lieu de rencontre scientifique, mais aussi un lieu qui, dans les forums de discussion, fait montre d'une conscience accrue pour des questions professionnelles et politiques. Parmi les maîtres de conférence et les chargés de cours, la parité des sexes est pratiquement atteinte. Au niveau des professeurs titulaires d'une chaire, cela reste encore à faire. Contrairement au manque d'encadrement des doctorants et post-doctorants encore déploré en 1997, on peut constater que, depuis, certaines universités suisses ont créé des programmes de recherche qui s'adressent directement aux jeunes chercheurs. Le Fonds national suisse subventionne sous le titre de Pro*Doc des programmes interuniversitaires pour les doctorants en sciences humaines ${ }^{6}$.

8 Sans doute, dans le cadre des colloques, les dé- bats répétés (dont le plus récent en 2004-05 à Lausanne sous le titre L'historien de l'art et ses partenaires) avec les représentants des fondations, des institutions culturelles nationales et des universités ont- ils joué un rôle pour l'avancement de ce processus positif. Dans ce contexte, une autre revendication formulée dans le colloque finira peut-être aussi par être exaucée à l'avenir : celle de créer plus de stages rémunérés et d'emplois-jeunes dans les musées. 
Concernant la relation entre université et musée, on peut noter que la moitié des diplômés en histoire de l'art est embauchée par les soixante-dix musées de la Suisse ${ }^{7}$. Cette tendance perdure et se reflète également dans les nombreux cursus de muséologie et de conservation proposés par les universités, et les différents centres de formation au niveau des deuxième et troisième cycles. De même, les présentations thématiques des colloques annuels témoignent de cet intérêt en favorisant des sujets relatifs au musée (institutions, marché de l'art, expositions ${ }^{8}$. Le colloque est aussi l'occasion de familiariser les jeunes historiens de l'art avec des domaines professionnels moins connus et de leur conférer plus de popularité. Des participants issus de différents champs de recherche s'y retrouvent, forment des réseaux et créent ainsi des synergies au niveau scientifique. Afin d'intensifier ces processus et de donner aussi aux étudiants et aux petits groupes de travail l'accès aux informations et aux discussions, trois représentants du corps intermédiaire des départements d'histoire de l'art des universités de Berne et de Fribourg ont fondé en 2003 l'Association suisse pour la relève en histoire de l'art sous le nom d'articulations. Financée par des sources privées, l'association s'occupe d'une liste de diffusion proposant des informations spécialement conçues pour la relève. Elle organise une à deux réunions de travail par an et gère actuellement, à travers de petits groupes de travail, la mise en réseau international de la relève d'une part, et la réalisation d'un Guide en ligne de l'étudiant suisse en histoire de l'art d'autre part. Ce guide publie des adresses utiles et des modèles de CV et donne des renseignements sur des bourses, des cours d'été, des programmes d'échange et des cursus universitaires.

de favoriser l'échange et la circulation des informations dans un petit pays comme la Suisse est dû à la diversité et à la décentralisation dont le pays témoigne par ses frontières cantonales et linguistiques, ses différents systèmes et traditions universitaires. Cette situation particulière nécessite donc des vecteurs d'unification. Dans le même temps, cette diversité explique le succès des colloques pour jeunes chercheurs. Malgré leur déroulement à peu près standardisé, les rencontres bénéficient d'année en année de nouvelles initiatives, d'enthousiasme et de professionnalisme. On fait appel à des chercheurs invités et organise des débats sur des questions politiques ou scientifiques d'actualité.

L'échange scientifique révèle la part active de la Suisse dans l'évolution internationale de la discipline et des problématiques méthodologiques entre théorie et pratique. Le colloque permet de constater une tendance qui reflète la situation internationale : les questions relatives aux médias et à la sociologie dominent les discussions, tandis que les approches théoriques et pratiques des nouveaux médias et des sujets d'art contemporain sont de plus en plus favorisés au détriment des études médiévales et Renaissance et Baroque. Ces changements sont justifiés aussi bien par la médiatisation de l'art que par les données actuelles du marché du travail et par la nécessité scientifique et politique de légitimer la recherche. Dans cette même lignée, les colloques, au lieu de rassembler des projets de recherche très hétérogènes, s'inscrivent davantage dans une volonté de donner des directions thématiques claires, afin d'augmenter le niveau des discussions (comme notamment la table ronde sur « le rôle des nouveaux médias en histoire de l'art» en 1998 à Berne, les problématiques interdisciplinaires évoquées dans Canons : œuvres, processus, discours en 2005 à Berne et Image/ Espace en novembre 2006 à Zurich). Contrairement au vaste panorama initial, cette orientation vers une conception thématique (aussi large soit-elle) des colloques répond également à la demande faite par les organismes d'aide financière, même si cela 
risque de limiter les approches individuelles de la recherche au lieu de les soutenir. Reste à espérer que les colloques de la relève préservent leur diversité sans céder à la pression générale de se restreindre à des sujets à la mode, porté par des intérêts économiques. Ce sont les futures générations de chercheurs qui seront certainement préoccupées par l'actualité et la discussion critique de ces sujets.

\section{NOTES}

1. Dominique Radrizzani, Sylvie Wuhrmann, La soupe et les nuages, (colloque, Lausanne, 10-12 avril 1997), Lausanne 1999, p. 5.

2. Une présentation de tous les colloques et de leurs travaux se trouve sur http:// articulations.ch/index.php?page=fr/colloques_fr.php.

3. Philippe Lüscher, Véronique Mauron, Dominique Radrizzani, Catherine Raemy-Berthod, Sylvie Wuhrmann, «Résolutions du premier colloque de la relève suisse en histoire de l'art. Université de Lausanne, 10-12 avril 1997 », dans D. Radrizzani, S. Wuhrmann, cité n. 1, p. 305-308 ; voir aussi http://www.articulations.ch/pdf/Resolution.pdf.

4. Virginie Babey, Julia Gelshorn, Claude-Alain Kuenzi, Thomas Schmutz, Tristan Weddigen, «Theorie der Praxis - Praxis der Theorie. Historische Problemfelder und aktuelle Strategien im Museums- und Ausstellungsbetrieb », dans Thésis. Cahier d'histoire des collections et de muséologie, 4, 2004, p. 7-10.

5. De tels résultats ont déjà fait partie d'un rapport publié en 1996 par le conseil scientifique suisse et d'autres institutions. Il a été confirmé par une prise de position critique de l'ASHHA: Claude Lapaire, Marc-Joachim Wasmer, Evaluation der geisteswissenschaftlichen Forschung in der Schweiz. Grundlagenbericht für die Kunstgeschichte (Forschungspolitik, 33), Berne, 1996. Voir aussi Oskar Bätschmann, «Selbstkritik der Geisteswissenschaften », dans Die Geisteswissenschaften heute und morgen, Berne, 2002, p. 43-56, et Pascal Griener, "La science et les sciences humaines aujourd'hui - une futilité indispensable », dans ibid., p. 57-66.

6. http://www.fns.ch.

7. Monika Stucky-Schürer, «Berufschancen der Kunsthistoriker. Auswertung einer Umfrage der Vereinigung der Kunsthistoriker in der Schweiz », dans Unsere Kunstdenkmäler, 32, 1981, p. 511518.

8. Voir dans ce contexte surtout la publication des contributions du colloque à Neuchâtel: V. Babey et al., cité n. 4 ; voir également Stefanie Christ, Regula Crottet, "Theorie und Praxis in Neuenburg - Ein Bericht über das VII. Nachwuchskolloquium für Kunstgeschichte in der Schweiz ", dans Kunst + Architektur in der Schweiz, LV, 2004, p. 66-67.

9. http://www.articulations.ch. 
INDEX

Keywords : research, study, university, museum

Mots-clés : recherche, étude, université, musée

Index géographique : Suisse

Index chronologique : 2000

AUTEUR

JULIA GELSHORN

Centre allemand d'histoire de l'art/Université de Zurich, gelshorn@gmx.net 